\title{
Evaluación Genética de un Plantel de Cuyes Reproductores de Genotipo Perú
}

\author{
Genetic Evaluation of a Flock of Breeding Guinea Pigs of Peru Genotype \\ Elmer Meza R. ${ }^{1,4}$, Jorge Raymondi C. ${ }^{2}$, Sonia Cisneros A. ${ }^{3}$
}

\section{Resumen}

\begin{abstract}
El presente estudio tuvo por objetivo la evaluación genética de un grupo de animales del plantel de cuyes reproductores de genotipo Perú pertenecientes a la Estación Experimental Agraria Canaán del Instituto Nacional de Innovación Agraria de Ayacucho, Perú, para características de peso vivo al beneficio (PB) y velocidad de crecimiento (VC). Se empleó la información productiva y de parentesco de 162 cuyes, además del sexo, tipo de parto, mes de nacimiento y peso de la madre al parto, cuyo análisis de incidencia sobre las variables estudiadas se efectuó mediante un modelo de efectos fijos. La estimación de los valores de cría se realizó a través de un modelo animal-BLUP unicarácter, el cual incluyó como efectos fijos a aquellos factores ambientales sistemáticos de incidencia significativa, además de los efectos aleatorios derivados de los factores genéticos de los animales. Los componentes de (co)varianza incluidos en el modelo y los algoritmos computacionales fueron resueltos usando el software VCE4 v. 4.2 y PEST v. 4.0, respectivamente. Los valores de cría estimados para PB y VC alcanzaron desvíos de -84.56 a $79.28 \mathrm{~g}$ y de 1.39 a $-1.26 \mathrm{~g}$ /día, mientras que sus niveles de precisión variaron de $28.81 \mathrm{a}$ $89.37 \%$ y 28.46 a $88.35 \%$, respectivamente. La alta dispersión de los valores de crías y la elevada correlación genética entre los caracteres evaluados, permitió identificar animales con un alto mérito genético, ameritando su uso potencial como padres de la siguiente generación.
\end{abstract}

Palabras clave: cuy, modelo animal, valor de cría, precisión

\footnotetext{
${ }^{1}$ Escuela Profesional de Medicina Veterinaria, Facultad de Ciencias Agrarias, Universidad Nacional San Cristóbal de Huamanga, Ayacucho, Perú

${ }^{2}$ Estación Experimental Agraria Canaán, Instituto Nacional de Innovación Agraria, Ayacucho, Perú

${ }^{3}$ Municipalidad Provincial de Huamanga, Ayacucho, Perú

${ }^{4}$ E-mail: elzar2112@hotmail.com
}

Recibido: 12 de agosto de 2016

Aceptado para publicación: 18 de diciembre de 2016 
This study aimed to genetically evaluate part of the breeding population of guinea pigs, Peru genotype, of the Experimental Station of Instituto Nacional de Innovación Agraria, Ayacucho, Peru, for two parameters: body weight at slaughter (WL) and growth rate (SG). Productive data of of the animals and their family members were collected ( $n=162$ animals), plus environmental factors such as sex, type of birth, month of birth and parturition weight of the mother. The statistical significance on the studied variables was conducted by a fixed effects model. The estimation of breeding values of the offspring wad done by the animal model-BLUP unicharacter. The model included fixed effects that had statistical significance plus the derivative random effects of animal genetic factors. The components of (co) variance included in the model and computational algorithms were solved using the software VCE4 v. 4.2, and PEST v. 4.0, respectively. The estimated breeding values for WLand SG ranged from -84.56 to $79.28 \mathrm{~g}$ and from 1.39 to $-1.26 \mathrm{~g}$ /day, while their levels of accuracy ranged from 28.81 to $89.37 \%$, and 28.46 to $88.35 \%$ respectively. The high dispersion of breeding values of the offspring and the high genetic correlation between the traits allow the identification of animals of high genetic merit, and making possible their selection as parents for the next generation.

Key words: guinea pig, animal model, breeding value, accuracy

\section{INTRODUCCIÓN}

El cuy doméstico (Cavia porcellus), es una especie que tiene particular potencial carnicero debido a su prolificidad y la exquisitez de su carne (Chauca, 2007). Sin embargo, su producción es aun deficitaria en términos de calidad y cantidad, siendo su bajo grado de mejoramiento genético una de las principales causas, a razón de la obsolescencia de los procedimientos tradicionalmente usados en su mejoramiento en varias partes del país. Consecuentemente, los individuos usados como reproductores, al ser carentes de información objetiva respecto a su verdadera capacidad productiva, generan incertidumbre y pobres respuestas en los niveles productivos (Alenda y Bejar, 1995).

El mejoramiento genético se logra mediante la identificación de individuos genéticamente superiores que posteriormente son usados como padres de la siguiente generación (Cardellino y Rovira, 1987; Verde, 2002), pues es con base a la estimación precisa de su valor de cría que se seleccio- nan como reproductores (Alenda y Bejar, 1995; Verde, 2002). Entre los métodos existentes para inferir los valores de cría de los animales, se encuentran el mejor predictor lineal (BLP), la predicción insesgada lineal óptima (BLUP) basados en modelos mixtos, así como los métodos basados en la verosimilitud y para respuestas categóricas, y los procedimientos bayesianos; entre otros (Gianola, 2001; Gutiérrez, 2010). En la actualidad, muchas de las técnicas mencionadas son potenciadas con técnicas moleculares para asistir en la selección e identificación de individuos genéticamente superiores (López et al., 2007).

La metodología del modelo mixto o valoración BLUP, es uno de los métodos más utilizados para predecir el valor genético de los animales de granja (Alenda y Bejar, 1995; Gutiérrez, 2010). Esta metodología se basa en el uso de modelos matemáticos y procedimientos estadísticos que permiten estimar en forma simultánea los efectos ambientales y genéticos que producen la observación (Mrode, 1996; Cameron, 1997); asimismo, permite evaluar y estimar los valores 
genéticos de padres, madres, descendientes y demás antecesores directos e indirectos, puesto que combina toda la información fenotípica y de parentesco, tanto por vía paterna como materna (Henderson, 1984; Gutiérrez, 2010).

El presente estudio tuvo por objetivo valorar genéticamente a los cuyes con categoría de padres, madres y descendencia que forman parte del plantel de reproductoras de genotipo Perú de la Estación Experimental Agraria (EEA) Canaán del Instituto Nacional de Innovación Agraria (INIA) de Ayacucho, a través del uso de la metodología BLUP-modelo animal.

\section{Materiales Y Métodos}

El estudio se realizó en los ambientes de cría del programa de investigación en animales menores del EEA Canaán del INIA-Ayacucho, a mediados de 2009. Se evaluaron 162 animales de un total de 198 inicialmente identificados aleatoriamente, excluyéndose del análisis a aquellos individuos que murieron antes de la expresión del carácter, o que mostraron inconsistencia, y/o pérdida de información. Los datos usados para la estimación de los valores de cría procedió de los registros de producción de 91 descendientes de un total de 41 madres y 30 padres identificados.

El análisis de incidencia de los efectos fijos identificados sobre las variables peso vivo al beneficio (PB) y velocidad de crecimiento (VC), se realizó por medio de un modelo de efectos fijos y el uso del software SAS v. 8 (SAS institute, 2000). Los efectos fijos de incidencia significativa fueron incluidos en un modelo operacional mixto bajo la forma de un modelo animal unicarácter, puesto que incorporó al animal como efecto aleatorio, además del error (micro ambiente).
$\mathrm{Y}=\beta \mathrm{X}+\alpha \mathrm{Z}+\mathrm{e}$

donde:

$\mathrm{X}=$ Matriz de efectos fijos conocidos de incidencia significativa (sexo, tipo de parto y mes de nacimiento de la cría)

$Z=$ Matriz de efectos aleatorios conocidos (padres, madres y descendencia)

$\beta=$ Vector de incidencia de efectos ambientales conocidos

$\alpha=$ Vector de valores genéticos aditivos conocidos

$e=$ Error aleatorio (microambiente)

La heredabilidad del carácter PB y VC, considerados en el modelo operacional fue de 0.32 y 0.28 , respectivamente, siendo estimados sobre la base de los datos colectados en el presente estudio y la determinación de componentes de varianza por máxima verosimilitud restringida (REML), cuyo módulo se encuentra incorporado en el software VCE 4.2.5 (Groeneveld, 1998).

Los valores genéticos predichos $(\alpha)$ y los efectos ambientales $(\beta)$ fueron obtenidos a través del predictor BLUP (mejor predictor lineal insesgado) (Henderson, 1984; Mrode, 1996). La resolución de los algoritmos matemáticos conteniendo la base de datos productivos y genealógicos fue realizada por medio del software PEST 3.1 (Groeneveld et al., 1998).

\section{Resultados y Discusión}

En las figuras 1 y 2 se muestran los desvíos de valores de cría predichos de los 162 animales evaluados para los caracteres de peso vivo al beneficio (PB) y velocidad de crecimiento (VC), respectivamente. El rango de los desvíos de valores de cría para PB fue de -84.56 g a $79.29 \mathrm{~g}$, encontrándose además una mayor proporción de animales con valores de cría negativos (90), frente a los que obtuvieron valores positivos (72). Por otro lado, los desvíos de valores de cría para $\mathrm{VC}$, 


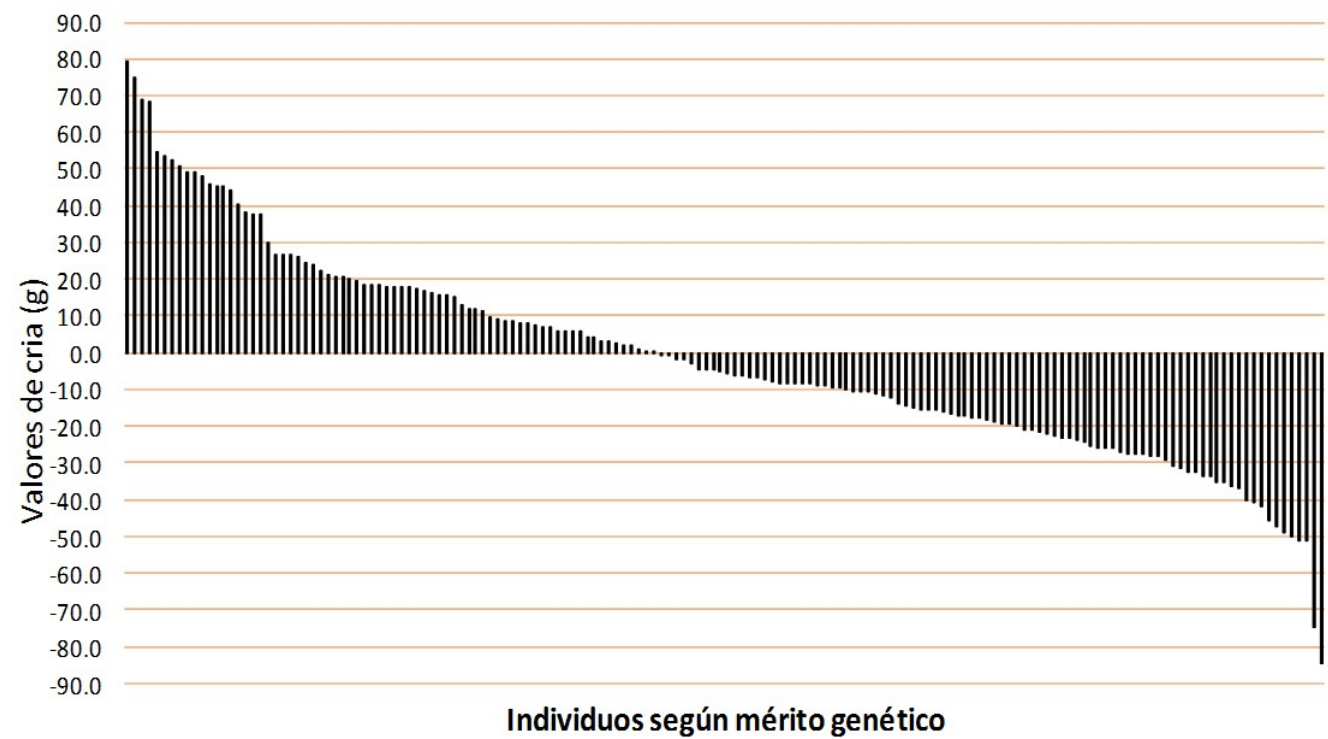

Figura 1. Valores de cría de los animales evaluados para el carácter peso vivo al beneficio (g) y ordenados según mérito genético

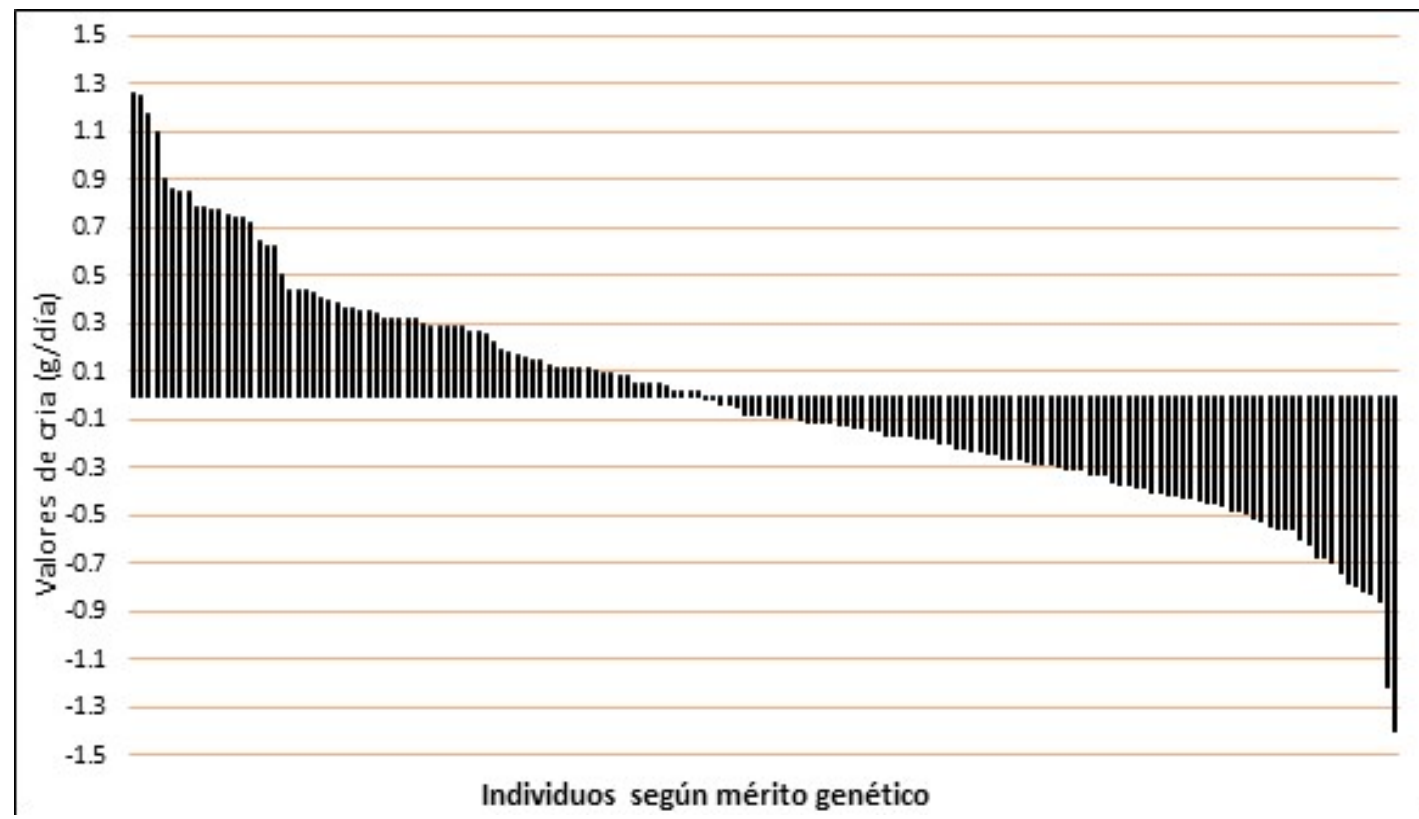

Figura 2. Valores de cría de los animales evaluados para el carácter velocidad de crecimiento (g/día) y ordenado según mérito genético 
registraron un rango de -1.39 a $1.26 \mathrm{~g}$ /día, encontrándose una mayor proporción de animales con valores de cría negativos (89) frente a los de valores de cría positivos (73).

Cabe mencionar que la mayor proporción de animales con valores de cría negativos lograron registrar mayores niveles de desvíos en sus magnitudes. Este hecho podría estar evidenciando una ineficacia de los procedimientos de mejoramiento tradicionalmente usados dentro del plantel de cuyes evaluado, lo que a su vez podría estar implicando un progreso genético no significativo de ambos caracteres; incluso, no podría descartarse la ausencia de progreso genético, así como una posible tendencia genética negativa de los caracteres evaluados. Sin embargo, resulta pertinente manifestar que los resultados del presente estudio no pretenden ser concluyentes, en vista de que solo se llegó a evaluar el 24\% $(n=162)$ de la población de cuyes de genotipo Perú del plantel, quedando la posibilidad de existir individuos con mayores méritos genéticos, y que probablemente estarían contribuyendo a la mejora genética de la población.

Entre los animales de mayor mérito genético se encontraron machos y hembras procedentes de camadas de varios tamaños, $\mathrm{y}$ pertenecientes a diferentes generaciones (tanto padres como descendientes). Esta particularidad se debe a que dichos individuos resultaron ser comparables desde el punto de vista genético, debido a que los factores ambientales que tuvieron incidencia significativa en la expresión del carácter $(\mathrm{p}<0.05)$ lograron ser controlados mediante la conformación de grupos contemporáneos, además del uso de un modelo operacional apropiado $\mathrm{y}$ un predictor de efectos fijos y genéticos (Gianola, 2001; Solarte et al., 2002). A su vez, los padres y madres de los 92 descendientes, a los cuales se les hizo el seguimiento de su desempeño productivo, si bien no lograron registrar datos productivos propios, tuvieron relación filial de parentesco con su descen- dencia, lo cual permitió estimar sus respectivos valores de cría, aunque con un menor nivel de precisión (Henderson, 1984; Mrode, 1996).

Los niveles de precisión resultaron ser similares en los dos caracteres bajo estudio, puesto que no difirieron significativamente en la magnitud de sus respetivos índices de herencia. Por tanto, con heredabilidades de 0.32 para el carácter peso al beneficio y de 0.28 para el carácter velocidad de crecimiento, se lograron niveles de precisión de 29.0 a $90.0 \%$ y de 28.7 a $89.1 \%$, respectivamente; siendo menores en aquellos animales que pertenecieron a la población base y que tuvieron un menor número de descendencia, en relación a aquellos que contaron con información propia, además de un mayor número de parientes colaterales (hermanos).

Finalmente, se constató que el orden de mérito de los animales evaluados, en función a la magnitud de sus valores de cría, mantuvo una misma correspondencia para los dos caracteres estudiados. Esto implica que los animales que ocuparon los primeros puestos para el carácter peso edad al beneficio, también lo fueron para el carácter velocidad de crecimiento. Este aspecto se debe a la alta correlación positiva de origen genético aditivo existente entre ambos caracteres (Cerón, 1998; Rodríguez, 2013). En consecuencia, la selección unicarácter centrado en el peso vivo al beneficio, sería suficiente para lograr el cambio genético favorable del carácter velocidad crecimiento.

\section{Conclusiones}

- Los valores de cría estimados para los caracteres de peso vivo al beneficio (g) y velocidad de crecimiento (g/día) alcanzaron desvíos de -84.56 a $79.28 \mathrm{~g}$ y de 1.39 a - 1.26 g/día, respectivamente; encontrándose una mayor proporción de animales con valores de cría negativos. 
- Los niveles de precisión de los valores de cría estimados tuvieron un rango de 28.81 a $89.37 \%$ y 28.46 a $88.35 \%$ para los caracteres de peso vivo al beneficio y velocidad de crecimiento, respectivamente; siendo mayor en individuos que registraron información productiva propia y en sus parientes colaterales.

\section{Literatura Citada}

1. Alenda R, Bejar F. 1995. Predicción del valor genético. Métodos. En: Zootecnia. Bases de producción Animal. Tomo 4, Cap. 6. Genética, patología, higiene y residuos animales. Madrid: Mundi-prensa. p 15-112.

2. Cameron ND. 1997. Prediction of breeding values and effects. In: Selection indices and prediction of genetic merit in animal breeding. Edinburgh, UK: CAB international. p 122-134.

3. Cardellino R, Rovira J. 1987. Mejoramiento genético animal. Montevideo: Ed Hemisferio Sur. $253 \mathrm{p}$.

4. Cerón MF, Córdoba OA, Solarte CA, Delgado DE. 1998. Genetic and phenotypic parameters of some characteristics of economic importance in guinea pigs Cavia porcellus. En: $6^{\text {th }}$ World Congress on Genetics Applied to Livestock Production. Armidale, NSW Australia.

5. Chauca FL. 2007. Logros obtenidos en la mejora genética del cuy (Cavia porcellus). Experiencias del INIA. Arch Latinoam Prod Anim 15: 218-222.

6. Gianola D. 2001. Los métodos estadísticos en el mejoramiento genético. University of Wisconsin-Madison, USA. [Internet]. Disponible en: http:// blog.utp.edu.co/genetica/files/2015/09/ genetic_improvement.pdf

7. Groeneveld E. 1998. VCE4.2.5 User's guide and reference manual, v. 1.1. Institute of Animal Husbandry and Ani- mal Behavior, Federal Agricultural Research Center, Germany.

8. Groeneveld E, Kovac M, Wang T. 1998. Multivariate prediction and estimation (PEST), v. 3.1. Department of Animal Sciences, University of Illinois.

9. Gutiérrez JP. 2010. Iniciación a la valoración genética animal. Metodología adaptada al EEES. España: UCM Ed Complutense. $355 \mathrm{p}$.

10. Henderson CR. 1984. Applications of linear models in animal breeding. University of Guelph, Ontario, Canada. [Internet]. Available in: ftp:// tech.obihiro.ac.jp/suzuki/Henderson.pdf

11. López ZR, Cano CH, Chassin NO, Zavala PM. 2007. Selección asistida por marcadores genéticos moleculares en especies animales de interés pecuario. Cienc Nicolaita 46: 43-55.

12. Mrode RA. 1996. Linear models for the prediction of animal breeding values. UK: CAB International. $\mathrm{p} 187$.

13. Rodríguez H. 2013. Evaluación fenotípica y estimación de parámetros genéticos en características productivas y reproductivas en cuyes de la granja de Cieneguilla. Tesis doctoral. Lima: Univ Nacional Agraria La Molina. $79 \mathrm{p}$.

14. SAS Institute. 2000. The SAS system for Windows, user's guide. Statistic v. 8.0. Cary, NC: Edition SAS Inst.

15. Solarte C, Imuez AM, Pérez T. 2002. Modelo animal multicarácter para la estimación de parámetros genéticos del Cavia porcellus en Colombia. $\mathrm{Rev} \mathrm{Cu}-$ bana Cienc Agríc 36: 19-24.

16. Verde O. 2002. De bakewell al modelo animal en la selección de reproductores. Conferencia. En: Memorias XI Congreso Venezolano de Producción e Industria Animal. Trujillo, Venezuela. [Internet]. Disponible en: http:// www.venezuelaganadera.com/enciclopedia-ganadera-articulos/de-bakewellal-modelo-animal-en-la-seleccion-dereproductores 